\title{
GLOBAL SURJECTIVITY OF SUBMERSIONS VIA CONTRACTIBILITY OF THE FIBERS
}

\author{
PATRICK J. RABIER
}

\begin{abstract}
We give a sufficient condition for a $C^{1}$ submersion $F: X \rightarrow Y$, $X$ and $Y$ real Banach spaces, to be surjective with contractible fibers $F^{-1}(y)$. Roughly speaking, this condition "interpolates" two well-known but unrelated hypotheses corresponding to the two extreme cases: Hadamard's criterion when $Y \simeq X$ and $F$ is a local diffeomorphism, and the Palais-Smale condition when $Y=\mathbb{R}$. These results may be viewed as a global variant of the implicit function theorem, which unlike the local one does not require split kernels. They are derived from a deformation theorem tailored to fit functionals with a norm-like nondifferentiability.
\end{abstract}

\section{INTRODUCTION}

If $X$ and $Y$ are real Banach spaces and $F: X \rightarrow Y$ is a $C^{1}$ submersion, i.e. $F^{\prime}(x)$ is onto $Y$ for every $x \in X$, are there conditions ensuring that $F$ is onto $Y$ ? And when $F$ is surjective, what can be said about the topological structure of the "fibers" $F^{-1}(y), y \in Y$ ? From a 1967 work of Earle and Eells [8] we get this answer to the second question:

Theorem 1.1. Let $X$ and $Y$ be real Banach spaces and $F: X \rightarrow Y a C^{1}$ submersion. Assume that

(i) $F$ is onto $Y$.

(ii) $\operatorname{ker} F^{\prime}(x)$ splits for $x \in X$.

(iii) For every $x \in X, F^{\prime}(x)$ has a right inverse $s(x) \in \mathscr{L}(Y, X)$ such that the mapping $s: X \rightarrow \mathscr{L}(Y, X)$ is locally Lipschitz continuous and $s(x) /(1+\|x\|)$ is locally bounded over $Y\left({ }^{1}\right)$ i.e. for every $y_{0} \in Y$ there are a neighborhood $V_{0}$ of $y_{0}$ in $Y$ and $\eta_{0}>0$ such that $\|s(x)\| /(1+\|x\|) \leq$ $\eta_{0}, \forall x \in F^{-1}\left(V_{0}\right)$.

Then, $F: X \rightarrow Y$ is a locally trivial $C^{0}$ fiber bundle. In particular, $F^{-1}(y)$ is contractible for $y \in Y$, and any two fibers are homeomorphic.

Earle and Eells' method of proof of Theorem 1.1 is as follows: assumptions (i), (ii) and (iii) are used to establish the bundle structure by a horizontal lifting of paths. Local Lipschitz continuity is involved via an associated ODE, and local boundedness is needed for the completeness of the flow. This part extends

Received by the editors August 29, 1994.

1991 Mathematics Subject Classification. Primary 58C15; Secondary 58B20, 58B05, 55R 10.

This work was supported in part by ONR-grant N-00014-90-J-1025.

${ }^{1}{ }^{1}$ In [8], it is assumed that $s$ is locally bounded over $Y$, but this generalization is straightforward. 
to $C^{1}$ Banach manifolds with a Finsler structure. Contractibility of $F^{-1}(y)$ is obtained through its status of Banach manifold, the homotopy sequence, and a theorem of J.H.C. Whitehead.

The major trouble with Theorem 1.1 is that its conditions (i) and (iii) are hard to check in concrete applications, even for finite dimensional $X$ and $Y$. Regarding (i), surjectivity, results are scarce for mappings which do not enjoy any particular properness property. On the other hand, proper submersions, which are open and closed, are surjective. But as we shall see, they are nothing more than diffeomorphisms except perhaps in some rather pathological cases (Corollary 4.3). This implies the failure of the usual method of proof of Hadamard's theorem to establish surjectivity instead of bijectivity, and explains the apparent absence of such results from the literature. As for condition (iii) of Theorem 1.1 , the difficulty comes from local boundedness over $Y$, which holds for proper maps (not too useful in our setting, as just mentioned), is accessible to verification in Hilbert spaces, but is generally out of reach in Banach spaces because there is no absolute constant bounding the norm of projections onto arbitrary complemented subspaces ([6] and [10]). We also note that, in practice, local Lipschitz continuity of the right inverse demands local Lipschitz continuity of $F^{\prime}$, i.e. $F$ to be of class $C^{2-}$ (in Palais' notation [12]). In particular, Theorem 1.1 does not apply to general $C^{1}$ submersions.

It is the aim of this paper to prove, by a quite different and direct method, a variant of Theorem 1.1 which is more readily and more widely applicable, and which in addition guarantees surjectivity of $F$ without any properness assumption (Theorem 4.1). More precisely, we shall only assume that a simple inequality holds, which in its crudest form reads

$$
\left\|F^{\prime}(x)^{*} y^{*}\right\| \geq c\left\|y^{*}\right\|, \quad \forall x \in X, \quad \forall y^{*} \in Y^{*},
$$

where $F^{\prime}(x)^{*} \in \mathscr{L}\left(Y^{*}, X^{*}\right)$ is the adjoint of $F^{\prime}(x)$ and $c>0$ is a constant, and that the space $Y$ has a norm of class $C^{1}$ away from the origin. This condition is of course satisfied when $Y$ is a Hilbert space, and also (Restrepo [14]) when the dual $Y^{*}$ of $Y$ is separable (whence $Y$ is separable). The sharper form of (1.1) we shall use is given in (4.2), and a variant of it corresponds to a generalization of the Palais-Smale condition for Banach space valued mappings (Corollary 4.1).

In all cases, surjectivity of $F$ and contractibility of the "fibers" $F^{-1}(y)$ will be ensured, although in our setting there is no guarantee that $F: X \rightarrow Y$ is a fiber bundle, as we are not assuming that $\operatorname{ker} F^{\prime}(x)$ splits for $x \in X$, or that $F$ is smoother than $C^{1}$. However, based upon Theorem 1.1, we prove that it is so if $X$ and $Y$ are Hilbert spaces and $F$ is of class $C^{2-}$ (Theorem 4.2). We also clarify the relationship between our condition (4.2) and the hypotheses of Theorem 1.1: if $\operatorname{dim} Y<\infty$, condition (4.2) is strictly weaker, and it is neither weaker nor stronger if $\operatorname{dim} Y=\infty$. In Corollary 4.2, we observe that Theorem 4.1 contains as a special case an improved version of Hadamard's theorem, which seems to supercede the other available variants. In this respect, it should be pointed out that when $F$ is a local diffeomorphism, (1.1) is equivalent to Hadamard's condition $\left\|F^{\prime}(x)^{-1}\right\| \leq k$, with $k=1 / c$. Finally, in Theorem 4.3 we show that the mappings satisfying the condition (4.2) also satisfy a converse of the intermediate value property: the inverse image of a connected open subset is connected. 
Everything hinges upon a deformation theorem (Theorem 3.1) which is the object of Sections 2 and 3. To establish contractibility, our approach makes no use of liftings or algebraic topology. It is very close to the methods of critical point theory, where the goal is to deform one level set into another in the absence of critical points. One major difference is that the classical theory applies only to functionals of class $C^{1}$ in the whole space $X$, while ours applies only to functionals which are not: for our assumptions to make sense, the functionals must behave like the absolute value function $|x|$ when $X=\mathbb{R}$, i.e. have a derivative bounded away from 0 in the vicinity of minimizers (this statement should not be taken literally; see Sections 2 and 3 for details). In Section 4, we use the deformation theorem with the functional $\|F-y\|, y \in Y$ fixed, to prove nonemptyness and contractibility of $F^{-1}(y)$.

For clarity of exposition, the simplest form of the deformation theorem, which suffices when (1.1) holds, is proved in Section 2, Corollary 2.1. Section 3 presents the final generalizations involving extra technicalities that we have preferred to keep separated so as not to obscure the issue.

As mentioned earlier, the main result in [8] extends to Banach manifolds with a Finsler structure. In contrast, ours remains valid only when $X$ but not $Y$ is such a manifold, because the need for a norm-like function of class $C^{1}$ on $Y$ rules out the cases when $Y$ is not (diffeomorphic to) a Banach space.

The deformation theorem (Theorem 3.1) has other applications, notably to critical point theory and to the structure of the set of minimizers of functionals, which will be presented elsewhere.

\section{A RESUlt OF CONTRACTIBILITY}

Let $X$ be a real Banach space and $J: X \rightarrow(-\infty, 0]$ a continuous functional. Denote by $Z$ the level set $J^{-1}(0)$. We shall assume throughout this section that $J$ is of class $C^{1}$ in $X \backslash Z$ and that there is a constant $c>0$ such that

$$
\left\|J^{\prime}(x)\right\| \geq c, \quad \forall x \in X \backslash Z \text {. }
$$

Obviously, (2.1) never holds if $Z \neq \varnothing$ and $J$ is $C^{1}$ in $X$, unless $J \equiv 0$, and hence nondifferentiability of $J$ is essential here.

Recall that a pseudo-gradient vector field for $J$ in $X \backslash Z$ is a locally Lipschitz continuous mapping $V: X \backslash Z \rightarrow X$ such that

$$
\|V(x)\| \leq 2\left\|J^{\prime}(x)\right\|, \quad \forall x \in X \backslash Z,
$$

and

$$
J^{\prime}(x) V(x) \geq\left\|J^{\prime}(x)\right\|^{2}, \quad \forall x \in X \backslash Z .
$$

By (2.1), we have $J^{\prime}(x) \neq 0, \forall x \in X \backslash Z$, and hence there is a pseudo-gradient vector field $V$ for $J$ in $X \backslash Z$ (see e.g. [12]). From (2.1), (2.2) and (2.3) it easily follows that

$$
\|V(x)\| \geq c, \quad \forall x \in X \backslash Z,
$$

and that

$$
\frac{J^{\prime}(x) V(x)}{\|V(x)\|} \geq \frac{c}{2}, \quad \forall x \in X \backslash Z
$$


For $x \in X \backslash Z$, let $\varphi(t, x) \in X \backslash Z$ denote the solution of the initial value problem

$$
\left\{\begin{array}{l}
\frac{d \varphi}{d t}(t, x)=\frac{V(\varphi(t, x))}{\|V(\varphi(x, t))\|} \\
\varphi(0, x)=x
\end{array}\right.
$$

Since $V$ is locally Lipschitz continuous in $X \backslash Z$, the same thing is true of $V /\|V\|$, whence $\varphi(t, x)$ is well-defined for $t$ in some maximal interval $[0, a(x))$ with $0<a(x) \leq \infty$. In the following lemma, we collect a few properties of the semi-flow $\varphi$ which are either trivial or well known from ODE theory.

Lemma 2.1. (i) Let $x \in X \backslash Z$ and $0 \leq t_{1} \leq t_{2}<a(x)$. Then

$$
\left\|\varphi\left(t_{2}, x\right)-\varphi\left(t_{1}, x\right)\right\| \leq t_{2}-t_{1}
$$

and

$$
J\left(\varphi\left(t_{2}, x\right)\right)-J\left(\varphi\left(t_{1}, x\right)\right) \geq \frac{c}{2}\left(t_{2}-t_{1}\right) .
$$

(ii) Let $x \in X \backslash Z$ and $0<T<a(x)$. For every $\epsilon>0$, there is $\delta>0$ such that

$$
\|y-x\|<\delta \Rightarrow\left\{T<a(y) \text { and } \sup _{t \in[0, T]}\|\varphi(t, x)-\varphi(t, y)\|<\epsilon\right\} .
$$

Proof. (i) The inequality (2.7) follows from the fact that the field $V /\|V\|$ has norm 1 . For the proof of (2.8), write

$$
J\left(\varphi\left(t_{2}, x\right)\right)-J\left(\varphi\left(t_{1}, x\right)\right)=\int_{t_{1}}^{t_{2}}\left(\frac{d}{d t} J(\varphi(t, x))\right) d t
$$

and use (2.5). Part (ii) is the standard continuous dependence upon initial values.

Our second preliminary lemma is slightly more technical.

Lemma 2.2. For $x \in X \backslash Z$, the following properties hold:

(i) $a(x)<\infty$.

(ii) $\varphi(a(x), x):=\lim _{t \rightarrow a(x)^{-}} \varphi(t, x)$ exists and is in $Z$.

(iii) The inequalities (2.7) and (2.8) remain valid for $t_{2}=a(x)$.

Proof. (i) Suppose by contradiction that $a(x)=\infty$, so that (2.8) holds with $t_{1}=0$ and $t_{2}=t>0$ arbitrary. Letting $t \rightarrow \infty$, we find $\lim _{t \rightarrow \infty} J(\varphi(t, x))=\infty$, contradicting the hypothesis $J \leq 0$.

(ii) Let $t_{n} \in[0, a(x))$ be a sequence such that $\lim _{n \rightarrow \infty} t_{n}=a(x)$. From (i), $\left(t_{n}\right)$ is a Cauchy sequence, whence $\varphi\left(t_{n}, x\right)$ is a Cauchy sequence by (2.7). Furthermore, if $\tilde{t}_{n} \in[0, a(x))$ is another sequence such that $\lim _{n \rightarrow \infty} \tilde{t}_{n}=a(x)$, it follows from (2.7) that $\lim _{n \rightarrow \infty}\left\|\varphi\left(t_{n}, x\right)-\varphi\left(\tilde{t}_{n}, x\right)\right\|=\lim _{n \rightarrow \infty}\left|t_{n}-\tilde{t}_{n}\right|=0$, so that $\lim _{n \rightarrow \infty} \varphi\left(t_{n}, x\right)$ is independent of the sequence $\left(t_{n}\right)$, i.e. $\lim _{t \rightarrow a(x)^{-}} \varphi(t, x)$ exists.

If $\lim _{t \rightarrow a(x)^{-}} \varphi(t, x) \in X \backslash Z$, then $\varphi(\cdot, x)$ can be extended to some interval $[0, a(x)+\epsilon)$ with $\epsilon>0$, in contradiction with the maximality of $[0, a(x))$ (recall $a(x)<\infty$ from part (i)). Thus, $\lim _{t \rightarrow a(x)^{-}} \varphi(t, x) \in Z$. 
(iii) In inequalities (2.7) and (2.8), let $t_{2}$ tend to $a(x)$. By (ii) above and continuity of $\|\cdot\|$ and continuity of the function $J$, we obtain the validity of (2.7) and (2.8) for $t_{2}=a(x)$.

We now extend $a$ and $\varphi$ to $X$ and $[0, \infty) \times X$, respectively, by setting

$$
a(x)=0, \quad \varphi(t, x)=x \text { if } x \in Z \text { and } t \geq 0
$$

and

$$
\varphi(t, x)=\varphi(a(x), x) \in Z \text { if } x \in X \backslash Z \text { and } t>a(x) .
$$

Note that the definition (2.10) makes sense in view of Lemma 2.2 (ii).

Theorem 2.1. The above extensions of $a$ and $\varphi$ are continuous.

Proof. We begin with continuity of $a$ in $X \backslash Z$. Part (ii) of Lemma 2.1 already ensures that $a$ is lower semicontinuous in $X \backslash Z$. To prove upper semicontinuity of $a$, suppose by contradiction that there are a sequence $x_{n} \in X \backslash Z$ with $\lim _{n \rightarrow \infty} x_{n}=x \in X \backslash Z$ and $\eta>0$ such that $a\left(x_{n}\right)>a(x)+\eta, \forall n \in \mathbb{N}$.

Choose $r>0$ such that $r<c \eta / 4$ (with $c$ as in (2.1)). In (2.8), let $t_{2}=a(x)$ (see Lemma 2.2 (iii)) and $t_{1}=T \in[0, a(x))$. Because $\varphi(a(x), x) \in Z$ and $\lim _{T \rightarrow a(x)^{-}} \varphi(T, x)=\varphi(a(x), x)$ (Lemma 2.2 (ii)) and because $J$ is continuous, we have $\lim _{T \rightarrow a(x)^{-}} J(\varphi(T, x))=0$. Thus, by choosing $T$ close enough to $a(x)$, we can manage so that $J(\varphi(T, x)) \geq-r$. From Lemma 2.1 (ii), we have $\lim _{n \rightarrow \infty} \varphi\left(T, x_{n}\right)=\varphi(T, x)$, whence $\lim _{n \rightarrow \infty} J\left(\varphi\left(T, x_{n}\right)\right)=J(\varphi(T, x))$. As a result, for $n$ large enough, we have

$$
J\left(\varphi\left(T, x_{n}\right)\right) \geq-2 r .
$$

Now, from (2.8) with $x_{n}$ replacing $x$ and $t_{1}=T, t_{2}=a(x)+\eta\left(<a\left(x_{n}\right)\right)$ we infer that

$$
J\left(\varphi\left(a(x)+\eta, x_{n}\right)\right) \geq J\left(\varphi\left(T, x_{n}\right)\right)+\frac{c}{2}(a(x)+\eta-T) .
$$

Using (2.11) and $a(x)-T>0, r<c \dot{\eta} / 4$, we find (for $n$ large enough)

$$
J\left(\varphi\left(a(x)+\eta, x_{n}\right)\right) \geq-2 r+\frac{c \eta}{2}>0,
$$

in contradiction with the hypothesis $J \leq 0$. This shows that $a$ is upper semicontinuous, hence continuous, in $X \backslash Z$.

We now pass to the proof of the continuity of the extension of $a$ defined by $a(x)=0$ for $x \in Z$. Clearly, it suffices to show that if $x \in Z$ and $x_{n} \in X \backslash Z, \lim _{n \rightarrow \infty} x_{n}=x$, then $\lim _{n \rightarrow \infty} a\left(x_{n}\right)=a(x)(=0)$. To see this, replace $x$ by $x_{n}$ and choose $t_{1}=0, t_{2}=a\left(x_{n}\right)$ in (2.8) (Lemma 2.2 (iii)). Since $\varphi\left(0, x_{n}\right)=x_{n}$ and $\varphi\left(a\left(x_{n}\right), x_{n}\right) \in Z=J^{-1}(0)$ ((2.6) and Lemma 2.2 (ii)), we obtain

$$
0<a\left(x_{n}\right) \leq-\frac{2}{c} J\left(x_{n}\right),
$$

which implies $0 \leq \lim _{n \rightarrow \infty} a\left(x_{n}\right) \leq-\frac{2}{c} J(x)=0$. 
To prove the continuity of the extension of $\varphi$ in (2.9) and (2.10), we choose $(t, x) \in[0, \infty) \times X$ and a sequence $\left(t_{n}, x_{n}\right) \in[0, \infty) \times X$ such that $\lim _{n \rightarrow \infty}\left(t_{n}, x_{n}\right)$ $=(t, x)$, and consider four cases.

Case 1: $0 \leq t<a(x), x \in X \backslash Z$. If so, we have $x_{n} \in X \backslash Z$ for $n$ large enough, and $\lim _{n \rightarrow \infty} \varphi\left(t_{n}, x_{n}\right)=\varphi(t, x)$ follows from Lemma 2.1 (ii).

Case 2: $t=a(x), x \in X \backslash Z$. Once again, $x_{n} \in X \backslash Z$ for $n$ large enough, and by definition (see (2.10)) we have $\varphi\left(t_{n}, x_{n}\right)=\varphi\left(a\left(x_{n}\right), x_{n}\right)$ if $t_{n}>a\left(x_{n}\right)$. Thus, replacing $t_{n}$ by $a\left(x_{n}\right)$ when $t_{n}>a\left(x_{n}\right)$, we still have $\lim _{n \rightarrow \infty} t_{n}=a(x)=t$ by continuity of $a$, and $\varphi\left(t_{n}, x_{n}\right)$ is unchanged. This shows that we may assume $t_{n} \leq a\left(x_{n}\right)$ with no loss of generality.

Let $0 \leq T<a(x)$ be arbitrary. From Lemma 2.1 (ii), $\varphi\left(T, x_{n}\right)$ is well defined for $n$ large enough and

$$
\lim _{n \rightarrow \infty} \varphi\left(T, x_{n}\right)=\varphi(T, x) .
$$

Next, by (2.7) with $t_{1}=T, t_{2}=a(x)$ (Lemma 2.2 (iii)), we find

$$
\|\varphi(a(x), x)-\varphi(T, x)\| \leq a(x)-T \text {. }
$$

Likewise, for $t_{n} \in\left[0, a\left(x_{n}\right)\right)$ as well as for $t_{n}=a\left(x_{n}\right)$ :

$$
\left\|\varphi\left(t_{n}, x_{n}\right)-\varphi\left(T, x_{n}\right)\right\| \leq\left|t_{n}-T\right| \text {. }
$$

Now, write

$$
\begin{aligned}
\left\|\varphi\left(t_{n}, x_{n}\right)-\varphi(a(x), x)\right\| \leq & \left\|\varphi\left(t_{n}, x_{n}\right)-\varphi\left(T, x_{n}\right)\right\|+\left\|\varphi\left(T, x_{n}\right)-\varphi(T, x)\right\| \\
& +\|\varphi(T, x)-\varphi(a(x), x)\| .
\end{aligned}
$$

Taking the limit as $n \rightarrow \infty$ and using (2.15), (2.16) and (2.17), we find (recall $\left.\lim _{n \rightarrow \infty} t_{n}=a(x)\right)$

$$
\varlimsup_{n \rightarrow \infty}\left\|\varphi\left(t_{n}, x_{n}\right)-\varphi(a(x), x)\right\| \leq 2(a(x)-T),
$$

whence $\varlimsup_{n \rightarrow \infty}\left\|\varphi\left(t_{n}, x_{n}\right)-\varphi(a(x), x)\right\|=0$ since $0 \leq T<a(x)$ is arbitrary. Equivalently, $\lim _{n \rightarrow \infty} \varphi\left(t_{n}, x_{n}\right)=\varphi(a(x), x)$.

Case 3: $t>a(x), x \in X \backslash Z$. Here, we have $x_{n} \in X \backslash Z$ and $t_{n}>a\left(x_{n}\right)$ (by continuity of $a$, proved above) for $n$ large enough. Thus (see (2.10)), $\varphi\left(t_{n}, x_{n}\right)=\varphi\left(a\left(x_{n}\right), x_{n}\right)$ and $\varphi(t, x)=\varphi(a(x), x)$, so that $\lim _{n \rightarrow \infty} \varphi\left(t_{n}, x_{n}\right)=$ $\varphi(t, x)$ if and only if $\lim _{n \rightarrow \infty} \varphi\left(a\left(x_{n}\right), x_{n}\right)=\varphi(a(x), x)$, which follows from Case 2 with $t_{n}=a\left(x_{n}\right)$.

Case 4: $x \in Z$. From (2.9), we have $\varphi(t, x)=x$, and $\varphi\left(t_{n}, x_{n}\right)=x_{n}$ if $x_{n} \in Z$. Thus, the only nontrivial part of the proof consists in showing that $\lim _{n \rightarrow \infty} \varphi\left(t_{n}, x_{n}\right)=x$ when $x_{n} \in X \backslash Z$. Furthermore, using once again $\varphi\left(t_{n}, x_{n}\right)=\varphi\left(a\left(x_{n}\right), x_{n}\right)$ if $t_{n}>a\left(x_{n}\right)$, it suffices to consider the case when $t_{n} \leq a\left(x_{n}\right)$.

Choosing $T=0$ in (2.17) (still valid), we see that $\left\|\varphi\left(t_{n}, x_{n}\right)-x_{n}\right\| \leq$ $t_{n} \leq a\left(x_{n}\right)$. By continuity of $a, \lim _{n \rightarrow \infty} a\left(x_{n}\right)=a(x)=0$ (see (2.9)). Thus, $\lim _{n \rightarrow \infty}\left\|\varphi\left(t_{n}, x_{n}\right)-x_{n}\right\|=0$, i.e. $\lim _{n \rightarrow \infty} \varphi\left(t_{n}, x_{n}\right)=x$. This completes the proof. 
Corollary 2.1. The set $Z=J^{-1}(0)$ is (nonempty and) contractible.

Proof. Let $a$ and $\varphi$ be extended to $X$ and $[0, \infty) \times X$, respectively, according to (2.9) and (2.10). From Theorem 2.1, these extensions are continuous. Define $\psi:[0,1] \times X \rightarrow X$ by $\psi(t, x)=\varphi(a(x) t, x)$, so that $\psi$ is continuous with $\psi(0, x)=x$ for $x \in X, \psi(1, x) \in Z$ for $x \in X$, and $\psi(t, x)=\varphi(0, x)=x$ for $x \in Z$ and $t \in[0,1]$. This means that $\psi$ is a (strong) deformation retraction of $X$ onto $Z$. It is both standard and elementary that this implies that $X$ and $Z$ have the same homotopy type (see e.g. [3]), and since $X$ is contractible, $Z$ is also contractible.

Remark 2.1. The (straightforward) argument used in the proof of Corollary 2.1 is needed in the case when $X$ is a Banach manifold. When $X$ is a Banach space, as assumed here, an explicit deformation of $Z$ (within itself) into a point is given by the mapping

$$
(t, z) \in[0,1] \times Z \longmapsto \varphi(a((1-t) z),(1-t) z) \in Z,
$$

which is identity for $t=0$ by (2.9), and constant $(=\varphi(a(0), 0))$ for $t=1$.

\section{Generalizations}

Our applications in the next section are based on generalizations of Corollary 2.1 involving sharpenings of Theorem 2.1 where now $c$ in (2.1) becomes a function of $x$. For clarity, we first consider two different cases separately in the next two lemmas.

Lemma 3.1. Theorem 2.1 and Corollary 2.1 remain valid if, in (2.1), we let $c=c(\|x\|)$ where $c:[0, \infty) \rightarrow(0, \infty)$ is a nonincreasing function such that $\int_{0}^{\infty} c(s) d s=\infty$.

Proof. Existence of $a$ (locally Lipschitz continuous) pseudo-gradient vector field $V$ for $J$ in $X \backslash Z$ depends only upon $J^{\prime}(x)$ being nonzero in $X \backslash Z$ and not upon (2.1). Thus, $V$ continues to exist and there is nothing to change in Section 2 up to and including inequality (2.7), if $c=c(\|x\|)$ everywhere. Evidently, inequality (2.8) makes no longer sense as stated, and should be replaced by

$$
J\left(\varphi\left(t_{2}, x\right)\right)-J\left(\varphi\left(t_{1}, x\right)\right) \geq \frac{1}{2} \int_{t_{1}}^{t_{2}} c(\|x\|+s) d s .
$$

To see that (3.1) holds, note that the method of proof of (2.8) first yields $J\left(\varphi\left(t_{2}, x\right)\right)-J\left(\varphi\left(t_{1}, x\right)\right) \geq \frac{1}{2} \int_{t_{1}}^{t_{2}} c(\|\varphi(s, x)\|) d s$. From (2.7) with $t_{2}=s$ and $t_{1}=0$, it follows that $\|\varphi(s, x)\| \leq\|x\|+s$, whence $c(\|\varphi(s, x)\|) \geq c(\|x\|+s)$ by monotonicity of $c$. This proves (3.1).

Aside from replacing (2.8) by (3.1) everywhere, the proofs of the previous section go through with only minor modifications, described below. To begin with, for the proof of Lemma 2.2 (i), it must be observed that $\int_{0}^{\infty} c(\|x\|+s) d s=$ $\infty$ because $\int_{0}^{\infty} c(s) d s=\infty$ and $\int_{0}^{\|x\|} c(s) d s<\infty$ by monotonocity of $c$.

Next, at the beginning of the proof of Theorem 2.1, $r>0$ should now be chosen such that $r<\frac{1}{4} \int_{a(x)}^{a(x)+\eta} c(M+s) d s$, where $M>0$ is a constant bounding 
the norm of the convergent sequence $\left(x_{n}\right)$. Inequality (2.12) should now read

$$
J\left(\varphi\left(a(x)+\eta, x_{n}\right)\right) \geq J\left(\varphi\left(T, x_{n}\right)\right)+\frac{1}{2} \int_{T}^{a(x)+\eta} c(M+s) d s .
$$

Relations (2.11) (unchanged) and (3.2), along with $T<a(x)$ thus yield, instead of (2.13)

$$
J\left(\varphi\left(a(x)+\eta, x_{n}\right)\right) \geq-2 r+\frac{1}{2} \int_{a(x)}^{a(x)+\eta} c(M+s) d s,
$$

and the choice of $r$ continues to provide the desired contradiction.

Lastly, inequality (2.14) should be replaced by

$$
0<\int_{0}^{a\left(x_{n}\right)} c(M+s) d s \leq-2 J\left(x_{n}\right)
$$

where once again $M>0$ is a constant bounding the norm of the convergent sequence $\left(x_{n}\right)$. If $a\left(x_{n}\right)$ does not tend to 0 , say $a\left(x_{n}\right) \geq \alpha>0$ after considering a subsequence, then (3.3) implies

$$
0<\int_{0}^{\alpha} c(M+s) d s \leq-2 J\left(x_{n}\right) .
$$

But $c(M+s) \geq c(M+\alpha)$ for $0 \leq s \leq \alpha$ by monotonocity of $c$, whence $0<$ $\alpha c(M+\alpha) \leq-2 J\left(x_{n}\right)$. As $J\left(x_{n}\right) \rightarrow 0$ and $\alpha>0$, this implies $c(M+\alpha)=0$, contradicting the positivity of $c$. Thus, as before, $\lim _{n \rightarrow \infty} a\left(x_{n}\right)=0$. No further modification is needed in the remainder of the proof of Theorem 2.1. That its validity implies that of Corollary 2.1 is obvious.

Theorem 2.1 (hence Corollary 2.1) cannot be improved much further beyond Lemma 3.1 if the constant $c$ in (2.1) is replaced by a function of $x$ involving only $\|x\|$. But if $c$ depends upon $x$ via $J(x)$, things go quite differently. This case is considered in the subsequent lemma.

Lemma 3.2. Theorem 2.1 and Corollary 2.1 remain valid if, in (2.1), we let $c=c(-J(x))$ where $c:[0, \infty) \rightarrow(0, \infty)$ is nonincreasing and lower semicontinuous.

Note: In sharp contrast with Lemma 3.1, there is here no limit about the rate of decay of $c$ at infinity.

Proof. Once again, except for replacing $c$ by $c(-J(x))$. everywhere, there is nothing to change in Section 2 up to and including inequality (2.7). In addition, even (2.8) remains valid with $c$ replaced by $c(-J(x))$. Indeed, from (2.5) with $c=c(-J(x))$ and $(2.6)$, it follows that $J(\varphi(\cdot, x))$ is increasing, whence $c(-J(\varphi(\cdot, x)))$ is nondecreasing, in [0,a(x)). Also from (2.5) and (2.6),

$$
J\left(\varphi\left(t_{2}, x\right)\right)-J\left(\varphi\left(t_{1}, x\right)\right) \geq \frac{1}{2} \int_{t_{1}}^{t_{2}} c(-J(\varphi(s, x))) d s,
$$

and the claim follows from $c(-J(\varphi(s, x))) \geq c(-J(x))$ for $s \in\left[t_{1}, t_{2}\right] \subset$ $[0, a(x))$. As a result, the proof of Lemma 2.2 goes through verbatim upon replacing $c$ by $c(-J(x))$ in (2.8). 
At the beginning of the proof of Theorem 2.1, choose $r>0$ such that $r<$ $c(-J(x)) \eta / 4$. Nothing else needs to be changed up to and including inequality (2.11) and, instead of (2.12), we now have

$$
J\left(\varphi\left(a(x)+\eta, x_{n}\right)\right) \geq J\left(\varphi\left(T, x_{n}\right)\right)+\frac{c\left(-J\left(x_{n}\right)\right)}{2}(a(x)+\eta-T) .
$$

By (2.11) and $a(x)-T>0$, this yields (for $n$ large enough)

$$
J\left(\varphi\left(a(x)+\eta, x_{n}\right)\right) \geq-2 r+c\left(-J\left(x_{n}\right)\right) \frac{\eta}{2} .
$$

Using continuity of $J$ and lower semicontinuity of $c$, we obtain

$$
\varliminf_{n \rightarrow \infty} J\left(\varphi\left(a(x)+\eta, x_{n}\right)\right) \geq-2 r+c(-J(x)) \frac{\eta}{2},
$$

and hence $\underline{\lim } J\left(\varphi\left(a(x)+\eta, x_{n}\right)\right)>0$ from the choice of $r$. This requires $J\left(\varphi\left(a(x)+\eta, x_{n}\right)\right)>0$ for $n$ large enough, in contradiction with the hypothesis $J \leq 0$. This proves continuity of $a$.

A final modification occurs in (2.14), which becomes

$$
0<a\left(x_{n}\right) \leq \frac{-2 J\left(x_{n}\right)}{c\left(-J\left(x_{n}\right)\right)} .
$$

Since $J\left(x_{n}\right) \rightarrow 0$ and $c$ is nonincreasing, we have $c\left(-J\left(x_{n}\right)\right) \geq c_{0}>0$ for every $n \in \mathbb{N}$, where $c_{0}$ is a constant. Thus,

$$
0<a\left(x_{n}\right) \leq-\frac{2}{c_{0}} J\left(x_{n}\right),
$$

showing, as before, that $\lim _{n \rightarrow \infty} a\left(x_{n}\right)=0$. The end of the proof of Theorem 2.1 remains the same.

Remark 3.1. In Lemma 3.2, monotonocity of $c$ is convenient for the proof (and will suffice for our applications) but actually irrelevant. Instead, one may use the fact that a lower semicontinuous function achieves its minimum value on every compact set.

The arguments used in the proofs of Lemmas 3.1 and 3.2 can easily be combined to yield Theorem 3.1 below, where a full statement is given for future reference and where $J$ has been changed into $-J$ to reinstate a more customary positivity (instead of negativity) assumption.

Theorem 3.1. Let $X$ be a real Banach space and $J: X \rightarrow[0, \infty)$ a continuous functional of class $C^{1}$ in $X \backslash Z$ where $Z:=J^{-1}(0)$. Suppose that there are nondecreasing functions $c_{1}, c_{2}:[0, \infty) \rightarrow(0, \infty)$ with $\int_{0}^{\infty} c_{1}(s) d s=\infty$ and $c_{2}$ lower semicontinuous, such that

$$
\left\|J^{\prime}(x)\right\| \geq c_{1}(\|x\|) c_{2}(J(x)), \quad \forall x \in X \backslash Z .
$$

Then, $Z \neq \varnothing$ is a contractible subset of $X$.

Note: Condition (3.5) cannot hold if $J$ is $C^{1}$ in $X$, unless $J \equiv 0$, for otherwise $Z \neq \varnothing$ from Theorem 3.1, and $J^{\prime}(z)=0$ for $z \in Z$. But then, (3.5) fails to hold for $x \in X \backslash Z$ close enough to $z$. 
Proof. Change $J$ into $-J$ to recover the setting of Section 2 and of Lemmas 3.1 and 3.2. By checking the proofs of these lemmas, it appears that except for replacing $c$ by $c_{1}(\|x\|) c_{2}(-J(x))$, nothing has to be changed in Section 2 up to and including inequality (2.7). It also appears that inequality (2.8) now takes the form

$$
J\left(\varphi\left(t_{2}, x\right)\right)-J\left(\varphi\left(t_{1}, x\right)\right) \geq \frac{c_{2}(-J(x))}{2} \int_{t_{1}}^{t_{2}} c_{1}(\|x\|+s) d s,
$$

which suffices for the validity of Lemma 2.2 .

At the beginning of the proof of Theorem 2.1, choose

$$
r<\frac{c_{2}(-J(x))}{4} \int_{a(x)}^{a(x)+\eta} c_{1}(M+s) d s
$$

where $M>0$ is a constant bounding the norm of the convergent sequence $\left(x_{n}\right)$. Combining the arguments of the proofs of Lemmas 3.1 and 3.2 we find, instead of (2.13), that

$$
\varliminf_{n \rightarrow \infty} J\left(\varphi\left(a(x)+\eta, x_{n}\right)\right) \geq-2 r+\frac{c_{2}(-J(x))}{2} \int_{a(x)}^{a(x)+\eta} c_{1}(M+s) d s,
$$

in contradiction with $J \leq 0$ for $n$ large enough from the choice of $r$.

Likewise, (2.14) becomes

$$
0<\int_{0}^{a\left(x_{n}\right)} c_{1}(M+s) d s \leq \frac{-2 J\left(x_{n}\right)}{c_{2}\left(-J\left(x_{n}\right)\right)} .
$$

Next, assuming by contradiction that $\lim _{n \rightarrow \infty} a\left(x_{n}\right) \neq 0$ and hence that $a\left(x_{n}\right) \geq$ $\alpha>0$ after considering a subsequence, we get

$$
0<\alpha c_{1}(M+\alpha) \leq \frac{-2 J\left(x_{n}\right)}{c_{0}}
$$

where $c_{0}>0$ is a constant such that $c\left(-J\left(x_{n}\right)\right) \geq c_{0}$. A contradiction with positivity of $c_{1}$ arises from $\alpha>0$ and $J\left(x_{n}\right) \rightarrow 0$. From this point on, the proof of Theorem 2.1 can be repeated without modifications, and of course Corollary 2.1 remains valid. Theorem 3.1 is proved.

As in Lemma 3.2, monotonocity of $c_{2}$ is not needed in Theorem 3.1 (see Remark 3.1). Obviously, the product $c_{1}(\|x\|) c_{2}(J(x))$ in (3.5) may be replaced by more general functions $c(\|x\|, J(x))$, but this does not seem to lead to substantial improvements in our applications.

More interesting is the (straightforward) extension of Theorem 3.1 to the case when $X$ is a complete $C^{2-}$ Banach manifold with a Finsler structure (see [12] or [7]), i.e. a complete Riemannian manifold when $\operatorname{dim} X<\infty$. The $C^{2-}$ requirement is needed for the existence of Lipschitz continuous pseudogradient vector fields. In this framework, $J^{\prime}(x)$ must be understood as the differential $d J(x)$, and the norm of $J^{\prime}(x)$ in (3.5) is that of $T_{x}^{*} X$, hence depends upon $x$. Naturally, it no longer makes sense to consider the function $c_{1}(\|x\|)$ since $X$ is not a vector space, but it can be replaced by $c_{1}\left(\delta\left(x, x_{0}\right)\right)$ where $x_{0} \in X$ is fixed and the distance $\delta$ is the Finsler metric of $X$. In this respect, note that in this form, (3.5) is independent of the choice of $x_{0}$ : 
given another point $\tilde{x}_{0} \in X$, we have $\delta\left(x_{0}, x\right) \leq \delta\left(x_{0}, \tilde{x}_{0}\right)+\delta\left(\tilde{x}_{0}, x\right)$, whence $c_{1}\left(\delta\left(x_{0}, x\right)\right) \geq c_{1}\left(\delta\left(x_{0}, \tilde{x}_{0}\right)+\delta\left(\tilde{x}_{0}, x\right)\right)$, and (3.5) holds with $\tilde{x}_{0}$ replacing $x_{0}$ and $c_{1}$ replaced by the function $c_{1}\left(\delta\left(x_{0}, \tilde{x}_{0}\right)+s\right)$ which remains nonincreasing with infinite integral in $(0, \infty)$. Another (crucial) point is that the conclusion is no longer that $Z \neq \varnothing$ is contractible but that $Z \neq \varnothing$ has the same homotopy type as $X$.

\section{SuRJeCtivity OF $C^{1}$ SUBMERSIONS}

We begin with a simple lemma, presumably not new.

Lemma 4.1. Let $Y$ be a Banach space with norm $\|\cdot\|$ of class $C^{1}$ away from the origin and set $N(y)=\|y\|, y \in Y$. Then,

$$
\left\|N^{\prime}(y)\right\|=1, \quad \forall y \in Y \backslash\{0\} .
$$

Proof. Let $y \in Y \backslash\{0\}$ be fixed. By positive homogeneity of $N$ ("Euler's theorem") we have $N^{\prime}(y) y=N(y)=\|y\|$, whence $\left\|N^{\prime}(y)\right\| \geq 1$. On the other hand, by convexity of $N$, the classical inequality

$$
N(z)-N(y) \geq N^{\prime}(y)(z-y), \quad \forall z \in Y,
$$

holds. Thus, for $v \in Y$, we have $N^{\prime}(y) v \leq\|y+v\|-\|y\| \leq\|v\|$, i.e. $\left\|N^{\prime}(y)\right\| \leq$ 1 .

Our "improved" (see Remark 4.1 later) version of Theorem 1.1 is as follows.

Theorem 4.1. Let $X$ and $Y$ be real Banach spaces such that $Y$ has a norm of class $C^{1}$ away from the origin, and let $F: X \rightarrow Y$ be a $C^{1}$ mapping. Suppose that for every sequence $\left(x_{n}\right)$ from $X$ the following condition holds

$$
F\left(x_{n}\right) \text { bounded } \Rightarrow \underline{\lim _{n \rightarrow \infty}}\left(1+\left\|x_{n}\right\|\right) \inf _{\left\|y^{*}\right\|=1}\left\|F^{\prime}\left(x_{n}\right)^{*} y^{*}\right\|>0\left(^{2}\right) \text {. }
$$

Then:

(i) $F(X)=Y$.

(ii) For every $y \in Y, F^{-1}(y)$ is closed in $X$ and contractible.

(iii) If $y \in Y$ and $\operatorname{ker} F^{\prime}(x)$ splits for every $x \in F^{-1}(y), F^{-1}(y)$ is a closed $C^{1}$ submanifold of $X$ (without boundary). Furthermore, $F^{-1}(y)$ is compact if and only if $F^{\prime}(x) \in G L(X, Y), \forall x \in F^{-1}(y)$, and in this case $F^{-1}(y)$ is a singleton.

(iv) If $\operatorname{ker} F^{\prime}(x)$ splits for every $x \in X$, the manifolds $F^{-1}(y), y \in Y$, are all modeled on the same closed subspace $E$ of $X$.

Proof. None of the assumptions is affected by changing $F$ into $F-y$ for some $y \in Y$. Accordingly, it suffices to show that $F^{-1}(0)$ is nonempty and contractible, and to prove part (iii) when $y=0$.

Set $J(x)=N(F(x))$ where, as in Lemma 4.1, $N$ denotes the norm of $Y$. The functional $J$ is continuous, of class $C^{1}$ in $X \backslash Z$ with $Z=J^{-1}(0)=$ $F^{-1}(0)$, and $J \geq 0$. Also, $J^{\prime}(x) h=\left\langle N^{\prime}(F(x)), F^{\prime}(x) h\right\rangle, h \in X$, where $\langle\cdot, \cdot\rangle$ denotes the duality pairing between $Y^{*}$ and $Y$, whence

$$
J^{\prime}(x)=F^{\prime}(x)^{*} N^{\prime}(F(x)), \quad x \in X \backslash Z .
$$

$\left({ }^{2}\right)$ In particular, $F$ is a submersion; see the proof of the theorem. 
From Lemma 4.1,

$$
\left\|J^{\prime}(x)\right\| \geq \inf _{\left\|y^{*}\right\|=1}\left\|F^{\prime}(x)^{*} y^{*}\right\|, \quad \forall x \in X \backslash Z,
$$

and hence condition (4.2) implies that for every sequence $\left(x_{n}\right)$ from $X \backslash Z$ we have

$$
J\left(x_{n}\right) \text { bounded } \Rightarrow \underset{n \rightarrow \infty}{\lim _{n \rightarrow \infty}}\left(1+\left\|x_{n}\right\|\right)\left\|J^{\prime}\left(x_{n}\right)\right\|>0 .
$$

In turn, it follows from (4.3) that for every $R>0$, there is $\gamma_{R}>0$ such that

$$
\{x \in X \backslash Z, J(x)<R\} \Rightarrow(1+\|x\|)\left\|J^{\prime}(x)\right\| \geq \gamma_{R} .
$$

In particular, letting $R=k \in \mathbb{N} \backslash\{0\}$, we obtain a sequence $\left(\gamma_{k}\right)$ of positive real numbers such that

$$
\{x \in X \backslash Z, J(x)<k\} \Rightarrow(1+\|x\|)\left\|J^{\prime}(x)\right\| \geq \gamma_{k} .
$$

Replacing $\gamma_{k+1}$ by $\min \left(\gamma_{k}, \gamma_{k+1}\right), k \geq 1$, we may assume that the sequence $\left(\gamma_{k}\right)$ is nonincreasing. For $s \in[0, \infty)$, set

$$
c_{2}(s)=\gamma_{j} \quad \text { if } s \in[j-1, j), j \in \mathbb{N} \backslash\{0\},
$$

so that $c_{2}$ is a positive nonincreasing lower semicontinuous function. Given $x \in X \backslash Z$, let $k \geq 1$ be the smallest integer such that $k-1 \leq J(x)<k$. Then, $c_{2}(J(x))=\gamma_{k}$ and, by (4.4)

$$
(1+\|x\|)\left\|J^{\prime}(x)\right\| \geq c_{2}(J(x)) .
$$

Now, for $s \in[0, \infty)$, set

$$
c_{1}(s)=1 /(1+s),
$$

whence $c_{1}$ is a positive decreasing function satifying $\int_{0}^{\infty} c_{1}(s) d s=\infty$. In this notation, (4.5) reads

$$
\left\|J^{\prime}(x)\right\| \geq c_{1}(\|x\|) c_{2}(J(x)), \quad \forall x \in X \backslash Z,
$$

and nonemptyness and contractibility of $Z$ follow from Theorem 3.1.

Condition (4.2) implies that $F$ is a submersion, for letting $x_{n}=x$ (constant sequence) we find $\inf _{\left\|y^{*}\right\|=1}\left\|F^{\prime}(x)^{*} y^{*}\right\|>0$, which implies that $F^{\prime}(x)$ is onto $Y$ (as is well known). Thus, if $\operatorname{ker} F^{\prime}(x)$ splits for $x \in Z, Z$ is a $C^{1}$ submanifold of $X$. Closedness of $Z$ in $X$ is clear.

For the "furthermore" part in (iii) of the theorem, note that $Z$ being modeled on the Banach space $E:=\operatorname{ker} F^{\prime}\left(x_{0}\right), x_{0} \in Z$ arbitrary, it is not locally compact, let alone compact, if $\operatorname{dim} E=\infty$. Suppose then that $\operatorname{dim} Z(=\operatorname{dim} E)=$ $p<\infty$. If $p=0$, we have $F^{\prime}(x) \in G L(X, Y)$ for $x \in Z$, and $Z$ is contractible of dimension 0 , hence a singleton. If $p \geq 1, Z$ cannot be compact, for compact contractible manifolds (without boundary) of positive dimension do not exist (see e.g. [1, p. 559]).

Part (iv) of the theorem is a general property of submersions with split kernels: by considering a splitting $\operatorname{ker} F^{\prime}\left(x_{0}\right) \oplus S_{0}$ where $S_{0}$ is a closed complement of $\operatorname{ker} F^{\prime}\left(x_{0}\right), x_{0} \in X$ fixed, it is easily seen that the restriction to $\operatorname{ker} F^{\prime}(x)$ of the projection onto $\operatorname{ker} F^{\prime}\left(x_{0}\right)$ is a linear isomorphism for $x$ close enough to $x_{0}$. Hence, the null-spaces $\operatorname{ker} F^{\prime}(x)$ are locally (in $X$ ) isomorphic. By compactness and connectedness of the line segment joining two arbitrary points in 
$X$, it follows at once that the null-spaces $\operatorname{ker} F^{\prime}(x), x \in X$, are all isomorphic. The conclusion follows from the fact that for $y \in Y, F^{-1}(y)(\neq \varnothing)$ is modeled on $\operatorname{ker} F^{\prime}(x)$ for any $x \in F^{-1}(y)$.

For practical purposes, note that $\inf _{\left\|y^{*}\right\|=1}\left\|F^{\prime}\left(x_{n}\right)^{*} y^{*}\right\|$ in (4.2) is obtained through

$$
\inf _{\left\|y^{*}\right\|=1}\left\|F^{\prime}(x)^{*} y^{*}\right\|=\inf _{\left\|y^{*}\right\|=1} \sup _{\|h\|=1}\left\langle y^{*}, F^{\prime}(x) h\right\rangle .
$$

Remark 4.1. It is of some interest to compare the hypotheses of Theorems 1.1 and 4.1: (1) When $\operatorname{dim} Y<\infty$, the hypotheses of Theorem 4.1 are weaker. In fact, condition (iii) of Theorem 1.1 alone implies (4.2). To see this, observe that local boundedness of $s(x) /(1+\|x\|)$ over $Y$ and finite dimensionality of $Y$ imply boundedness of $s(x) /(1+\|x\|)$ over the bounded subsets of $Y$, i.e. for $B \subset Y$, a bounded subset, there is $\eta(B)>0$ such that $\|s(x)\| /(1+\|x\|) \leq$ $\eta(B), \forall x \in F^{-1}(B)$. Then, for $x \in B$ and $y \in Y^{*},\left\|y^{*}\right\|=1$, we have

$$
\begin{gathered}
\sup _{h \in X \backslash\{0\}} \frac{\left\langle y^{*}, F^{\prime}(x) h\right\rangle}{\|h\|} \geq \sup _{v \in Y \backslash\{0\}} \frac{\left\langle y^{*}, F^{\prime}(x) s(x) v\right\rangle}{\|s(x) v\|}=\sup _{y \in Y \backslash\{0\}} \frac{\left\langle y^{*}, v\right\rangle}{\|s(x) v\|} \\
\geq \frac{1}{\eta(B)(1+\|x\|)} \sup _{v \in Y \backslash\{0\}} \frac{\left\langle y^{*}, v\right\rangle}{\|v\|}=\frac{1}{\eta(B)(1+\|x\|)}
\end{gathered}
$$

Thus, from (4.6), $(1+\|x\|) \inf _{\left\|y^{*}\right\|=1}\left\|F^{\prime}(x)^{*} y^{*}\right\| \geq 1 / \eta(B), \quad \forall x \in F^{-1}(B)$, which is (4.2) since $B$ is arbitrary. (2) When $\operatorname{dim} Y=\infty$, the hypotheses of Theorem 4.1 are no longer weaker: they would be only if " $F\left(x_{n}\right)$ bounded" could be replaced by " $F\left(x_{n}\right)$ convergent" in (4.2) (by an argument similar to the one used in (1) above) which of course is weaker when $\operatorname{dim} Y=\infty$, and an open question.

More can be said when $X$ and $Y$ are Hilbert spaces.

Theorem 4.2. Suppose that $X$ and $Y$ in Theorem 4.1 are Hilbert spaces. Then, condition (4.2) alone ensures the validity of (i), (ii), (iii) and (iv) of Theorem 4.1, and in addition we have

(v) If $F$ is of class $C^{2-}, F: X \rightarrow Y$ is a locally trivial $C^{\circ}$ fiber bundle, hence any two fibers $F^{-1}(y)$ are homeomorphic.

(vi) If $\operatorname{dim} X=\infty$ and $X$ is separable, hence $X \simeq \ell^{2}$, and $\operatorname{dim} \operatorname{ker} F^{\prime}\left(x_{0}\right)=$ $\infty$ for some $x_{0} \in X, F^{-1}(y)$ is $C^{\infty}$-diffeomorphic to $\ell^{2}$ for $y \in Y$.

Note: Observe that (vi) above holds with merely $F \in C^{1}$.

Proof. The first statement is obvious. We shall prove (v) by showing that Theorem 1.1 applies. Conditions (i) and (ii) of that theorem hold, the former by Theorem 4.1. Also, recall that condition (4.2) implies that $F$ is a submersion. Thus, it suffices to show that condition (iii) of Theorem 1.1 holds too.

We begin with a simple remark: if $A \in \mathscr{L}(X, Y)$ is onto $Y$, then rge $A^{*}$ $=(\operatorname{ker} A)^{\perp}$ (identifying $\left.X^{*}=X, Y^{*}=Y\right)$. Hence, for $h \in(\operatorname{ker} A)^{\perp} \backslash\{0\}$, there is $y \in Y \backslash\{0\}$ such that $h=A^{*} y$ and we have

$$
\frac{\|A h\|}{\|h\|}=\frac{\left\|A A^{*} y\right\|}{\left\|A^{*} y\right\|}=\frac{\left\|A A^{*} y\right\|\|y\|}{\left\|A^{*} y\right\|\|y\|} \geq \frac{\left(A A^{*} y, y\right)}{\left\|A^{*} y\right\|\|y\|}=\frac{\left\|A^{*} y\right\|}{\|y\|} .
$$


As a result, $\inf \left\{\|A h\|: h \in(\operatorname{ker} A)^{\perp},\|h\|=1\right\} \geq \inf _{\|y\|=1}\left\|A^{*} y\right\|$. Using this with $A=F^{\prime}(x), x \in X$, we find that (4.2) implies that for every bounded subset $B \subset Y$, there is a constant $\eta(B)>0$ such that

$$
(1+\|x\|) \inf \left\{\left\|F^{\prime}(x) h\right\|: h \in\left(\operatorname{ker} F^{\prime}(x)\right)^{\perp},\|h\|=1\right\}>\eta(B), \quad \forall x \in F^{-1}(B) .
$$

Next, let $x_{0} \in X$ be given, so that $F^{\prime}\left(x_{0}\right) \in G L\left(\left(\operatorname{ker} F^{\prime}\left(x_{0}\right)\right)^{\perp}, Y\right)$ and hence $F^{\prime}(x) \in G L\left(\left(\operatorname{ker} F^{\prime}\left(x_{0}\right)\right)^{\perp}, Y\right)$ for $x$ in some neighborhood $U_{0}$ of $x_{0}$ in $X$. Local Lipschitz continuity of $F^{\prime}$ implies local Lipschitz continuity of the right inverse

$$
s_{0}(x)=\left[F^{\prime}(x)_{\left.\right|_{\left(\operatorname{ker} F^{\prime}\left(x_{0}\right)\right)}}\right]^{-1} \in G L\left(Y,\left(\operatorname{ker} F^{\prime}\left(x_{0}\right)\right)^{\perp}\right), \quad x \in U_{0} .
$$

Now, for $x \in U_{0}$, the operator

$$
s(x)=\left[F^{\prime}(x)_{\left.\right|_{\left(\operatorname{ker} F^{\prime}(x)\right)^{\perp}}}\right]^{-1} \in \mathscr{L}(Y, X),
$$

is the product of the orthogonal projection $F^{\prime}(x)^{*}\left(F^{\prime}(x) F^{\prime}(x)^{*}\right)^{-1} F^{\prime}(x)$ onto $\left(\operatorname{ker} F^{\prime}(x)\right)^{\perp}$ and of $s_{0}(x)$ (the surjectivity of $F^{\prime}(x)$, ensured by (4.2), is equivalent to the invertibility of $\left.F^{\prime}(x) F^{\prime}(x)^{*}\right)$. Hence, $s$ above is locally Lipschitz continuous in $U_{0}$. But $s$ is defined everywhere in $X$, and $x_{0}$ is arbitrary, so that $s$ is locally Lipschitz continuous in $X$.

It remains to show that $s(x) /(1+\|x\|)$ is locally bounded over $Y$. Clearly,

$$
\|s(x)\|=1 / \inf \left\{\left\|F^{\prime}(x) h\right\|: h \in\left(\operatorname{ker} F^{\prime}(x)\right)^{\perp},\|h\|=1\right\} .
$$

Next, let $y_{0} \in Y$ be fixed, and let $V_{0}$ be any bounded neighborhood of $y_{0}$. From (4.7), there is $\eta_{0}:=\eta\left(V_{0}\right)>0$ such that

$$
(1+\|x\|) \inf \left\{\left\|F^{\prime}(x) h\right\|: h \in\left(\operatorname{ker} F^{\prime}(x)\right)^{\perp},\|h\|=1\right\} \geq \eta_{0}, \forall x \in F^{-1}\left(V_{0}\right),
$$

whence $\|s(x)\| /(1+\|x\|) \leq 1 / \eta_{0}$ for $x \in F^{-1}\left(V_{0}\right)$, as was to be proved.

For the proof of (vi), recall first that every separable $C^{1}$ Hilbert manifold may be equipped with a $C^{\infty}$ structure ([11]). In particular, this is true of $F^{-1}(y), y \in Y$, since $F^{-1}(y) \subset X$ and $X$ is separable. Next, any two separable Hilbert manifolds of infinite dimension having the same homotopy type are $C^{\infty}$ diffeomorphic (see [4, p. 380, footnote]). From the assumption $\operatorname{dim} \operatorname{ker} F^{\prime}\left(x_{0}\right)=\infty$ and Theorem 4.1 (iv), $F^{-1}(y)$ has infinite dimension for $y \in Y$. As $F^{-1}(y)$ is contractible by (ii) of the same theorem, $F^{-1}(y)$ has the same homotopy type as $\ell^{2}$, and the conclusion follows.

Remark 4.2. (1) Extra smoothness of $F$, e.g. $F \in C^{k+1}$, ensures that the fibration $F: X \rightarrow Y$ in part (v) of Theorem 4.2, is $C^{k}$ (see [8]), so that the fibers $F^{-1}(y)$ are $C^{k}$ diffeomorphic in this case. (2) In part (vi) of Theorem 4.2, separability of $X$ may be replaced by separability of the model $E:=\operatorname{ker} F^{\prime}\left(x_{0}\right), x_{0} \in X$. (3) Obviously, Theorem 4.2 (vi) is not true when $\operatorname{dim} \operatorname{ker} F^{\prime}(x)$ is finite (and hence the same for all $x \in X$ ), so that $F$ is Fredholm of index $p \geq 0$. We do not know when it can be ascertained that $F^{-1}(y)$ is diffeomorphic to $E=\mathbb{R}^{p}$ (the analog of Theorem 4.2 (vi) for this case), but we note that it can for $p=0,1$ and 2 . The result is trivial if $p=0$ since $F^{-1}(y)$ is a singleton (Theorem 4.1 (iii)). Likewise, if $p=1, F^{-1}(y)$ is diffeomorphic to $\mathbb{R}$, being a noncompact and connected one-dimensional manifold. For $p=2, F^{-1}(y)$ is a simply connected (because contractible) noncompact 
two-dimensional manifold, and those are known to be diffeomorphic to $\mathbb{R}^{2}$ ([9, p. 207]). For $p=4$, the result is unlikely to be true because of the existence of "fake" $\mathbb{R}^{4}$, but "homeomorphic" is plausible.

The following criterion is useful.

Corollary 4.1. In Theorem 4.1, condition (4.2) holds if $F$ is a submersion and every sequence $\left(x_{n}\right)$ from $X$ such that $F\left(x_{n}\right)$ is bounded and

$$
\lim _{n \rightarrow \infty}\left(1+\left\|x_{n}\right\|\right) \inf _{\left\|y^{*}\right\|=1}\left\|F^{\prime}\left(x_{n}\right)^{*} y^{*}\right\|=0
$$

contains a convergent subsequence (and hence no such sequence $\left(x_{n}\right)$ exists).

Proof. Suppose that the condition stated in Corollary 4.1 holds, and let $\left(x_{n}\right)$ be a sequence from $X$ such that $F\left(x_{n}\right)$ is bounded and

$$
\varliminf_{n \rightarrow \infty}\left(1+\left\|x_{n}\right\|\right) \inf _{\left\|y^{*}\right\|=1}\left\|F^{\prime}\left(x_{n}\right)^{*} y^{*}\right\|=0 \text {. }
$$

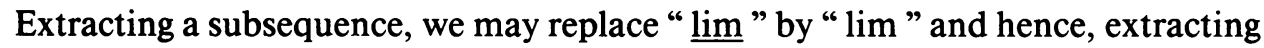
another subsequence, assume that

$$
\lim _{n \rightarrow \infty} x_{n}=x \text { and } \lim _{n \rightarrow \infty} \inf _{\left\|y^{*}\right\|=1}\left\|F^{\prime}\left(x_{n}\right)^{*} y^{*}\right\|=0 .
$$

To prove the corollary, we show that necessarily $\inf _{\left\|y^{*}\right\|=1}\left\|F^{\prime}(x)^{*} y^{*}\right\|=0$. Indeed, this contradicts surjectivity of $F^{\prime}(x)$ which, as is well known, implies the existence of a constant $\gamma>0$ such that $\left\|F^{\prime}(x)^{*} y^{*}\right\| \geq \gamma\left\|y^{*}\right\|, \forall y^{*} \in Y^{*}$.

For simplicity of notation, set $F^{\prime}\left(x_{n}\right)=A_{n}, F^{\prime}(x)=A$. By continuity of $F^{\prime}, \lim _{n \rightarrow \infty}\left\|A-A_{n}\right\|=0$, hence $\lim _{n \rightarrow \infty}\left\|A^{*}-A_{n}^{*}\right\|=0$. Now, $\inf _{\left\|z^{*}\right\|=1}\left\|A^{*} z^{*}\right\| \leq\left\|A^{*} y^{*}\right\| \leq\left\|A^{*}-A_{n}^{*}\right\|+\left\|A_{n}^{*} y^{*}\right\|, \quad \forall y^{*} \in Y^{*},\left\|y^{*}\right\|=1, \quad \forall n \in \mathbb{N}$.

As a result,

$$
\inf _{\left\|z^{*}\right\|=1}\left\|A^{*} z^{*}\right\| \leq\left\|A^{*}-A_{n}^{*}\right\|+\inf _{\left\|y^{*}\right\|=1}\left\|A_{n}^{*} y^{*}\right\|, \quad \forall n \in \mathbb{N},
$$

and the right-hand side tends to 0 , whence $\inf _{\left\|z^{*}\right\|=1}\left\|A^{*} z^{*}\right\|=0$, as desired.

When $Y=\mathbb{R}$, the condition in Corollary 4.1 coincides with the famous Palais-Smale condition $(C)$, in the weak form discovered by Cerami [5]. On the other hand, when $Y \simeq X$ and $F$ is a local diffeomorphism, we find

Corollary 4.2. Suppose that in Theorem 4.1,F is a local diffeomorphism. Then, $F$ is a $C^{1}$ diffeomorphism of $X$ onto $Y$. In particular, it is so if $F$ is a local diffeomorphism and for every sequence $\left(x_{n}\right)$ from $X$ we have

$$
F\left(x_{n}\right) \text { bounded } \Rightarrow \varlimsup_{n \rightarrow \infty} \frac{\left\|F^{\prime}\left(x_{n}\right)^{-1}\right\|}{1+\left\|x_{n}\right\|}<\infty
$$

Proof. Because $\left\|F^{\prime}(x)^{-1}\right\|=\left\|\left(F^{\prime}(x)^{*}\right)^{-1}\right\|$, we have

$$
\inf _{\left\|y^{*}\right\|=i}\left\|F^{\prime}(x)^{*} y^{*}\right\|=\frac{1}{\left\|F^{\prime}(x)^{-1}\right\|}
$$

i.e. (4.8) is equivalent to (4.2). Parts (i) and (iii) of Theorem 4.1 establish surjectivity and injectivity of $F$, respectively. 
We refer to [15] for other variants of Hadamard's theorem, which all follow from Corollary 4.2 (from the proof of Theorem 4.1, it is obvious that the weight $(1+\|x\|)^{-1}$ in $(4.8)$ could be replaced by $c(\|x\|)$ where $c:[0, \infty) \rightarrow(0, \infty)$ is nonincreasing and $\left.\int_{0}^{\infty} c(s) d s=\infty\right)$. Also, without " $F\left(x_{n}\right)$ bounded" in (4.8), the condition is known. As it stands, (4.8) is necessary (and sufficient) for the inverse $F^{-1}$ to have a derivative bounded on bounded subsets of $Y$ (hence necessary and sufficient when $\operatorname{dim} X=\operatorname{dim} Y<\infty)$. Indeed, if $\left(F^{-1}\right)^{\prime}$ maps bounded sets to bounded sets, we have $F^{\prime}\left(x_{n}\right)^{-1}=\left(F^{-1}\right)^{\prime}\left(F\left(x_{n}\right)\right)$ bounded whenever $F\left(x_{n}\right)$ is bounded, and (4.8) holds. For finite dimensional $X$ and $Y$, Corollary 4.2 can be further improved ([13]).

Because of Corollary 4.2, Theorem 4.1 may be viewed as a generalization of Hadamard's theorem, and it is natural to ask whether the properness criterion has a similar generalization, i.e. whether proper $C^{1}$ submersions are surjective. Submersions being open (even without split kernels, [1]) and proper mappings closed, the answer is positive. However, the usefulness of this remark depends partly upon the breadth of the class of proper submersions. Strong evidence that those are hardly more than diffeomorphisms has already been provided by Berger and Plastock [2], who showed that there is no $C^{2-}$ proper submersion which is Fredholm of index $p \geq 1$. Our next corollary goes a little bit further, in the same direction.

Corollary 4.3. Let $X$ and $Y$ be real Banach spaces such that $Y$ has a norm of class $C^{1}$ away from the origin and let $F: X \rightarrow Y$ be a proper $C^{1}$ submersion. If either

(i) $\operatorname{ker} F^{\prime}(x)$ splits for every $x \in X$ and $F$ is of class $C^{2-}$, or

(ii) $\operatorname{dim} Y<\infty$,

then $F$ is a diffeomorphism of $X$ onto $Y$.

Proof. In both cases (i) and (ii), $\operatorname{ker} F^{\prime}(x)$ splits for $x \in X$. As noticed above, $F$ is onto $Y$, whence $F^{-1}(y)$ is a $C^{1}$ submanifold of $X$ for $y \in Y$. Also, $F^{-1}(y)$ is compact so that $\operatorname{dim} F^{-1}(y)<\infty$ by an argument from the proof of Theorem 4.1 (iii). Since $\operatorname{dim} F^{-1}(y)=\operatorname{dim} \operatorname{ker} F^{\prime}(x), x \in F^{-1}(y)$, we have $\operatorname{dim} \operatorname{ker} F^{\prime}(x)<\infty, \forall x \in X$, i.e. $F$ is a Fredholm mapping with index $p \geq 0$.

In case (i) of the corollary, $p=0$ from the aforementioned result of Berger and Plastock. In case (ii), it follows from properness of $F$ and finite dimensionality of $Y$ that condition (4.2) holds, using the criterion of Corollary 4.1 (if $F\left(x_{n}\right)$ is bounded, it contains a convergent subsequence; next, use properness of $F$ ). By Theorem 4.1 (iii), we have $F^{\prime}(x) \in G L(X, Y), \forall x \in X$, so that, once again, $p=0$.

Thus, in both cases, $F$ is a proper local diffeomorphism (being a submersion), and hence a diffeomorphism of $X$ onto $Y$.

Remark 4.3. In sharp contrast with Corollary 4.3 , it is easy to find examples of mappings satisfying (4.2) which are not diffeomorphisms. For instance, all the linear continuous surjective maps do.

Our final result shows that the mappings of Theorem 4.1 satisfy a converse of the intermediate value property.

Theorem 4.3. Let $X$ and $Y$ be real Banach spaces such that $Y$ has a norm of class $C^{1}$ away from the origin, and let $F: X \rightarrow Y$ be a $C^{1}$ mapping satisfying 
condition (4.2) of Theorem 4.1. Then, the inverse image $F^{-1}(U)$ of an open connected subset $U \subset Y$ is (open and) connected.

Proof. As a first step, we show that the inverse image of a closed ball $\overline{B_{r}}$ with radius $r>0$, is contractible (the case considered in Theorem 4.1 is $r=0$ ). With no loss of generality, assume that $\overline{B_{r}}$ is centered at the origin, and for $x \in X$, set

$$
J_{r}(x)=\max (\|F(x)\|, r)-r .
$$

This functional is continuous, nonnegative and $Z_{r}:=J_{r}^{-1}(0)=F^{-1}\left(\overline{B_{r}}\right)$. For $x \in X \backslash Z_{r}$ we have $J_{r}(x)=\|F(x)\|-r=J(x)-r$ where $J$ is the functional of the proof of Theorem 4.1. Thus, $J_{r}$ is of class $C^{1}$ in $X \backslash Z_{r}$ and $J_{r}^{\prime}(x)=J^{\prime}(x)$ for $x \in X \backslash Z_{r}$. As was seen in the proof of Theorem 4.1, condition (4.2) implies that $J^{\prime}$ satisfies inequality (3.5) of Theorem 3.1 for $x \notin F^{-1}(0)$. As a result, $J_{r}^{\prime}$ satisfies the same inequality for $x \notin Z_{r}=F^{-1}\left(\overline{B_{r}}\right)$, and contractibility of $F^{-1}\left(\overline{B_{r}}\right)$ thus follows from Theorem 3.1.

Next, let $U \subset Y$ be a connected open subset, and let $x_{1}, x_{2} \in F^{-1}(U)$. We shall prove that there is a continuous path in $F^{-1}(U)$ joining $x_{1}$ to $x_{2}$, which suffices to establish its connectedness. Set $y_{i}=F\left(x_{i}\right) \in U, i=1,2$, and let $\sigma:[0,1] \rightarrow U$ be a continuous path joining $x_{1}$ to $x_{2}$. Since $U$ is open and $\sigma([0,1])$ is compact, the distance from $\sigma([0,1])$ to $Y \backslash U$ is positive, and hence there is $r>0$ such that the closed ball with center $\sigma(t)$ and radius $r$ is entirely contained in $U$ irrespective of $t \in[0,1]$. Consider a minimal (finite) covering of $\sigma([0,1])$ by the interiors $B_{i}, 1 \leq i \leq k$, of such balls. Using finiteness and minimality of the covering along with connectedness of $\sigma([0,1])$, it is easily seen that we may manage so that $y_{1} \in B_{1}$ and for $1 \leq i \leq k-$ 1, $\left(\bigcup_{j=1}^{i} B_{j}\right) \cap B_{i+1} \neq \varnothing$. Set $C_{i}=\bigcup_{j=1}^{i} \bar{B}_{j} \subset U$, so that $C_{i+1}=C_{i} \cup \bar{B}_{i+1}$ and $C_{i} \cap \bar{B}_{i+1} \neq \varnothing$. From the first part of the proof, $F^{-1}\left(\bar{B}_{i}\right)$ is contractible, hence pathwise connected. Since $F^{-1}\left(C_{1}\right)=F^{-1}\left(\bar{B}_{1}\right)$ and $F^{-1}\left(C_{i+1}\right)=F^{-1}\left(C_{i}\right) \cup$ $F^{-1}\left(\bar{B}_{i+1}\right), F^{-1}\left(C_{i}\right) \cap F^{-1}\left(\bar{B}_{i+1}\right) \neq \varnothing$, it follows by induction that $F^{-1}\left(C_{i}\right)$ is pathwise connected for $1 \leq i \leq k$. In particular, $F^{-1}\left(C_{k}\right)$ is pathwise connected, and $x_{1}, x_{2} \in F^{-1}(\sigma([0,1])) \subset F^{-1}\left(C_{k}\right) \subset F^{-1}(U)$. Thus, $x_{1}$ and $x_{2}$ can be joined by a continuous path in $F^{-1}\left(C_{k}\right)$, and therefore in $F^{-1}(U)$.

Remark 4.4. Naturally, more is true when $F: X \rightarrow Y$ is a locally trivial $C^{\circ}$ fiber bundle (e.g. when Theorem $4.2(\mathrm{v})$ applies), namely $F^{-1}(U)$ and $U$ have the same homotopy type for every open subset $U$ of $Y$. This follows from contractibility of $F^{-1}(y), y \in U$, and arguments from the proof of Theorem 1.1 .

From the remarks at the end of Section 3, it follows that the results of this section have counterparts when $X$ is a complete $C^{2-}$ Banach manifold with a Finsler structure (and $Y$ remains a Banach space with a $C^{1}$ norm away from the origin). Condition (4.2) must be modified into

$$
F\left(x_{n}\right) \text { bounded } \Rightarrow \underline{\lim _{n \rightarrow \infty}}\left(1+\delta\left(x_{n}, x_{0}\right)\right) \inf _{\left\|y^{*}\right\|=1}\left\|d F\left(x_{n}\right)^{*} y^{*}\right\|>0,
$$

where $\delta$ is the Finsler metric of $X, x_{0} \in X$ is fixed and $d F(x): T_{x} X \rightarrow Y$ is the differential of $F$ at $x$. Condition (4.10) is independent of the choice of $x_{0}$ (see Section 3). Contractibility of $F^{-1}(y)$ in part (ii) of Theorem 4.1 must be 
changed into " $F^{-1}(y)$ has the same homotopy type as $X$ " (and in particular is pathwise connected when $X$ is connected). For this reason, Corollary 4.3 is no longer valid as stated: if $X$ is the cylinder $S^{1} \times \mathbb{R}$, the projection $X=S^{1} \times$ $\mathbb{R} \rightarrow \mathbb{R}=Y$ is obviously a proper $C^{1}$ submersion but not a diffeomorphism. Similarly, in part (iii) of Theorem 4.1 , it can be ascertained that $F^{-1}(y)$ is not compact only if $X$ does not have the homotopy type of any compact manifold (without boundary). Theorem 4.2 (v) is unchanged, but in (vi) it is only true that $F^{-1}(y)$ and $F^{-1}(z)$ are $C^{\infty}$ diffeomorphic, unless $X$ is contractible (but then $X \simeq \ell^{2}$ from the arguments of the proof of Theorem 4.2 (vi), and hence that theorem applies). Theorem 4.3 remains valid if $X$ is connected (thus pathwise connected).

\section{ACKNOWLEDGMENTS}

This work was supported in part by ONR Grant N-00014-90-J-1025. I wish to thank J. Pejsachowicz for his always valuable comments and for mentioning to me the work of Berger and Plastock referenced in the text.

\section{REFERENCES}

1. R. Abraham, J.E. Marsden and T. Ratiu, Manifolds, tensor analysis and applications, 2nd ed., Springer-Verlag, New York, 1988.

2. M. S. Berger and R. A. Plastock, On the singularities of nonlinear Fredholm operators of positive index, Proc. Amer. Math. Soc. 76 (1980), 217-221.

3. G. E. Bredon, Topology and geometry, Springer-Verlag, New York, 1993.

4. D. Burghelea and N. M. Kuiper, Hilbert manifolds, Ann. of Math. 90 (1969), 379-417.

5. G. Cerami, Un criterio di esistenza per i punti critici su varietà illimitati, Rend. Acad. Sci. Lett. Istituto Lombardo 112 (1978), 332-336.

6. W. J. Davis, D. W. Dean and I. Singer, Complemented subspaces and $\Lambda$ systems in Banach spaces, Israel J. Math. 6 (1968), 303-309.

7. K. Deimling, Nonlinear functional analysis, Springer-Verlag, New York, 1985.

8. C. J. Earle and J. Eells, Foliations and fibrations, J. Differential Geom. 1 (1967), 33-41.

9. M. W. Hirsch, Differential topology, Springer-Verlag, New York, 1976.

10. J. Lindenstrauss and L. Tzafriri, On the complemented subspace problem, Israel J. Math. 9 (1971), 263-269.

11. N. Moulis, Approximation de fonctions differentiables sur certains espaces de Banach, Ann. Inst. Fourier 21 (1971), 293-345.

12. R. S. Palais, Lusternik-Schnirelman theory on Banach manifolds, Topology 5 (1966), 115132.

13. P. J. Rabier, On global diffeomorphisms of Euclidian space, Nonlinear Analysis, TMA 21 (1993), 925-947.

14. G. Restrepo, Differentiable norms in Banach spaces, Bull. Amer. Math. Soc. 70 (1964), 413-414.

15. E. Zeidler, Nonlinear functional analysis and its applications, Springer-Verlag, New York, 1985.

Department of Mathematics and Statistics, University of Pittsburgh, Pittsburgh, PenNSYlVania 15260

E-mail address: rabier@ums.cis.pitt.edu 